\title{
Automated 3D imaging at ambient and cryogenic conditions.
}

\author{
W.M. Busing, F. de Haas, U. Lucken, L. Melanson, W.M.A. Hax and Marc M.H. Storms. \\ FEI Company, Achtseweg Noord 5, 5651 GG Eindhoven, The Netherlands
}

Software intelligence to automate data acquisition is the key to results in modern microscopy. Imaging is no longer a 2D-projection of the 3D-structure of the specimen. The development of dedicated application software allows for automatic acquisition of series of images that can be subsequently processed into a 3D-representation of the specimen under investigation. Such 3Dtomogram is of paramount importance to create a better understanding of the complex threedimensional architecture of cells and cell-organelles.

Also Molecular Imaging has been transformed into a rapid, efficient, high throughput process leading to 3D structures of transient and stable molecular machines. These molecular machines are associations of individual components (proteins, nucleic acids, lipids) into large complexes; examples include ribosomes, transcription complexes and membrane embedded pumps and channels.

By using template-matching software, tomograms of frozen hydrated cells or cell organelles can be searched for the presence and location of particular molecular machines. In that way the cell biologist can unravel the supramolecular architecture and dynamics of the biological cell.

To preserve the native (hydrated) state of biological structures, FEI introduces the new Vitrobot mark III - a fully automated vitrification device for plunge-freezing of aqueous (colloidal) suspensions. The Vitrobot offers the unique opportunity to automate the cryo-fixation process at constant and user-definable physical and mechanical conditions (e.g. temperature, relative humidity, blotting conditions and freezing velocity). This ensures high quality cryo fixation results and a high sample preparation throughput prior to cryo observation in a TEM.

Once in the Tecnai microscope, the ultra-high and clean vacuum ensures a contamination free observation for long working sessions. In low temperature mode, the FEI microscopes can be used at both liquid nitrogen and helium temperatures. In combination with the Low Dose software this gives the optimal protection of beam sensitive specimens. Important other improvements made are the optimization of the low magnification high-resolution performance.

In this presentation, sample preparation and transfer, data acquisition and processing of results, including a description of the critical steps and factors for obtaining 3D-cryo results at either liquid nitrogen and/or helium temperatures, will be discussed.

\section{References}

[1] P. Frederik et al., B385, EUREM, Brno 2000.

[2] P. Zhang et al., J. of Struct. Biol. 135 (2001), 251.

[3] K. Grünewald et al., Science 302 (2003). 

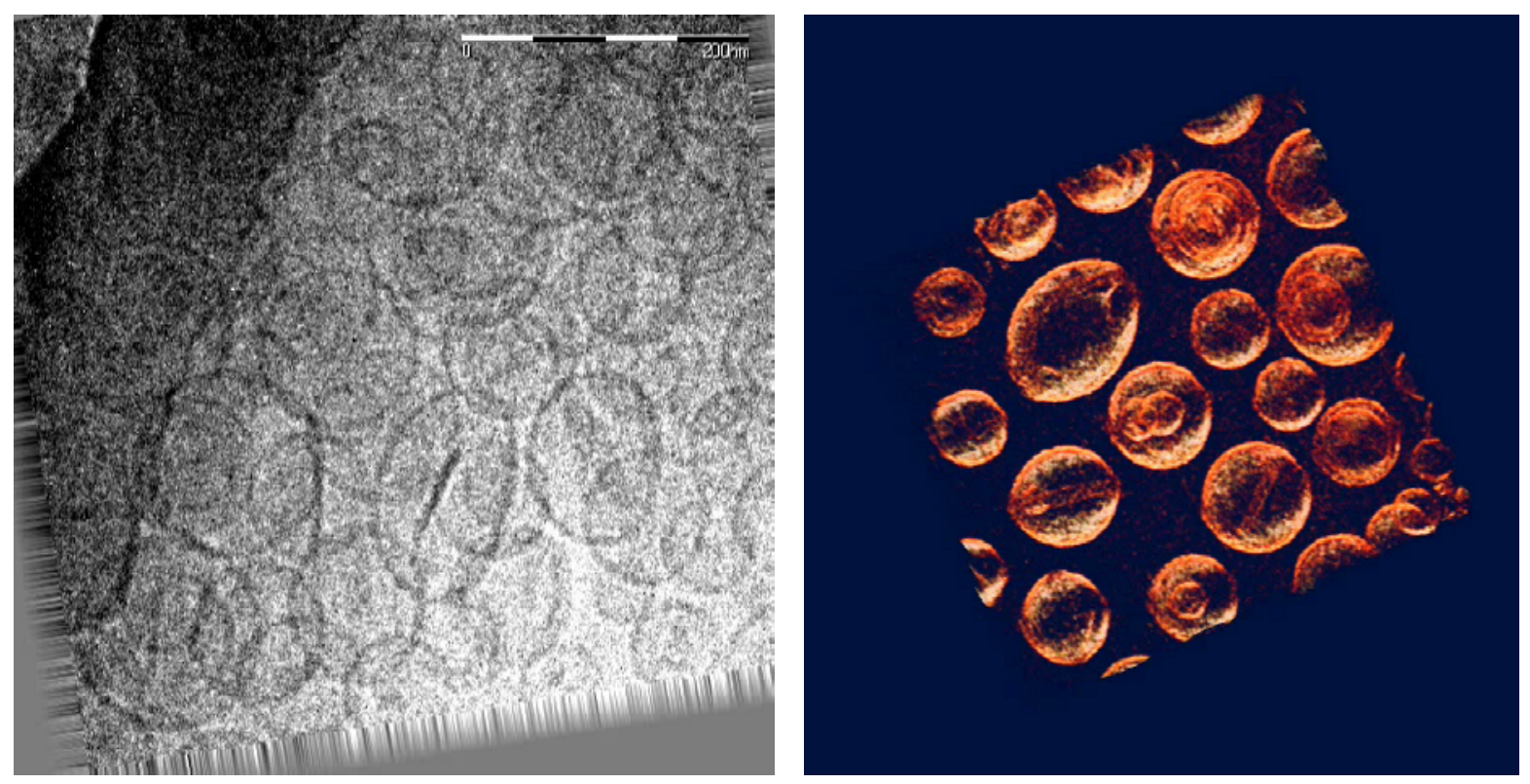

Fig. 1. Drug loaded liposomes - Image is Cryo-EM (15K) tomography of doxorubicin loaded vesicles recorded on Tecnai Polara, tilted $+/-50^{\circ}$ with 2 degrees increment, magnification 40,000x, total dose 140 e/Å2. Courtesy of Dr. Peter Frederik, EM Unit, University of Maastricht, The Netherlands. Specimen courtesy of Alza/Menlo Park CA 